\title{
Household Risk Factors and Intention of Safety Equipment Use among the Elderly: a Survey from Two Districts of Beijing
}

Qiujie Zhang, Yingnan Ma, Wei Zhu, Xing Gao, Pengxia Zhao*

Beijing Research Center of Urban Systems Engineering, Beijing 100035, China

\section{老年人居家风险因素及安全设备使用意愿分析 一一北京市月坛和羊坊店街道为例}

张秋洁, 马英楠, 朱伟, 高星, 赵鹏霞*

北京城市系统工程研究中心，北京 100875 , 中国

\begin{abstract}
To understand the household risk factors and willingness of safety equipment use of the elderly, 789 elderly people were surveyed with questionnaires in two districts of Beijing. Related factors in gender, age, level of education, physiological status, psychological status, household environment conditions and willingness of safety equipment use were analyzed. Results showed that risk factors generally existed among the elderly. Unsafe environmental conditions and lack of necessary safety equipment were major problems. Besides, compared with the elderly with fewer individual risk factors, the elderly with more risk factors were more likely to ignore their own safety status, and showed less use of safety equipment. It was suggested that the government should invest in environmental improvement in the districts according to the elderly people's safety needs. Related safety training and education should be provided to enhance the safety capacity of the elderly. Household environment conditions should be regularly checked to eliminate the hidden dangers. Safety equipment with high-level technology should be developed based on the needs of
\end{abstract}

*通讯作者：988656@qq.com the elderly. Special attention should be paid to the safety of the elderly with more individual risk factors.

Keywords: the elderly; risk factors; safety equipment use; living environmental conditions

\section{摘要}

为了解老年人居家风险因素及安全设备使用意愿, 减 少老年人居家安全伤害, 通过对北京市月坛和羊坊店 街道共 789 名老年人的问卷调查, 从性别、年龄、受 教育程度、生理因素、心理因素及居家环境等方面分 析了居家风险因素, 并对老年人的安全设备使用意愿 进行了分析。结果表明, 老年人普遍面临居家安全风 险, 其中, 居家环境存在不安全隐患、缺乏使用必要 的安全设备是较为突出的问题, 另外相较于没有个体 风险因素的老年人而言, 有个体风险因素的老年人往 往更容易忽视自身的安全, 更少使用安全设备。建议 老旧社区加强适老性改造, 加强对老年人安全的相关 培训和宣教工作, 提高老年人安全意识, 开展老年人 居住环境的隐患排查工作, 及时发现和消除环境安全 隐患。根据老年人需求, 研发科技水平高的安全设备, 满足老年人日常生活的需要, 重点关注个体风险高的 老年人。

关键词：老年人：风险因素；安全设备；居住环境 
Risk Analysis and Crisis Response in Big Data Era (RAC-16)

\section{1. 引言}

当前, 老年人伤害已经成为威胁老年健康和安全 的重大公共卫生问题[1]。我国已经步入老龄社会, 并正迎来第一个老龄高峰期。老年人由于年龄老化带 来的生理机能的衰退、控制环境能力下降、负心理感 受增强等, 成为伤害发生的高危人群和脆弱人群 [2], 伤害给老年人、家庭和社会都带来了沉重的负担[3]。 老年人伤害问题已经成为研究如何保护老年人安全、 促进老年人健康、提高老年人生活质量的关注点。由 于家庭是老年人活动的主要场所, 因此, 有必要对老 年人在家庭中面临的主要风险进行分析。

\section{2. 老年人伤害的风险因素研究}

随着老年人伤害的频发, 老年人风险因素分析及 伤害预防, 逐渐成为公共卫生领域关注和研究的重点 之一。国内外对于老年人伤害的因素分析, 一般归结 为: 生理因素、疾病和药物、社会心理及环境因素等。 2.1. 生理因素

随着年龄的增长, 生理机能减退是的老年人发生 伤害的重要危险因素。生理机能的减退使得老年人身 体虚弱, 日常生活能力受限制, 如身体协调性下降, 视觉、听觉及感知能力、反应能力减弱, 导致跌倒、 交通事故、烧汤伤、硬物碰撞等危险性增高[4-6]。此 外, 由于衰老长生的社会功能削弱、抑郁等, 会导致 负性不良情绪而使老年人出现自杀倾向[7]。

\section{2. 疾病和药物}

老年人慢性疾病和药物作用, 也被认为是伤害发 生的主要因素。Kerber[8]、刘丽丹[9]等人的研究均 表明, 各类老年疾病, 如高血压、冠心病、高血糖等 心脑血管疾病常伴有头晕、肢体障碍等, 增加了跌倒、 交通事故等风险。此外躯体疾病、精神疾病往往是老 人自杀的前提因素。MargdaWaern 的研究表明, 患有 躯体疾病的自杀老人占自杀老人综述的 $34 \%-94 \%[10]$; 抑郁症、精神分裂症等患者的自杀率 远高于一般群体。由于疾病等原因, 老年人服用药物 越多, 越容易受到药物副作用的影响, 进而导致感知、 情绪、及判断力受损, 增加了伤害发生的风险[11]。 2.3. 环境因素

环境因素也被认为是老年人伤害发生的直接原 因之一。多数的老年伤害发生在室内或社区周围。如 楼梯、门槛、地板，室内光线、家居摆设等均有可能 成为老年人跌倒、硬物撞击等伤害发生的直接因素 [12]; 此外, 高层建筑的电梯、消防安全也成为老年
人伤害发生的风险[13]。

\section{4. 社会心理因素}

社会心理因素也是老年人伤害发生的主要原因 之一。一方面, 由于年龄、生理的原因, 老年人面临 着退休、社会地位、家庭地位、社会关系等多重社会 心理的变化, 角色转变和 “突然失去” 往往给老年人 带来诸多的烦恼和压力, 加大了老年伤害的风险。另 一方面, 随着我国家庭结构和社会观念的转变, 独居、 空巢老年群体的增加, 老年人受到的家庭和照顾逐渐 减少, 社会支持的缺位和老年生活的孤独寂寞增加了 伤害发生的可能。

现有研究对老年人居家风险因素关注不多, 并且 较少关注老年人安全设备的使用情况。因此, 本调查 着重关注老年人的居家风险因素, 并对老年人安全设 备的使用情况及使用意愿进行了调查。

\section{3. 调查方法与数据}

\section{1. 调查对象}

本次调查选取了北京市的月坛街道和羊坊店街 道。月坛街道位于西城区, 羊坊店街道位于海淀区。 根据社区提供的信息, 月坛街道的总人口为 116543 人, 其中老年人 31997 人, 在月坛街道共发放问卷 450 份, 回收有效问卷 402 份; 羊坊店街道的总人口 为 127000 , 其中老年人 25400 人, 在羊坊店街道共 发放问卷 450 份, 回收有效问卷 387 份。

\section{2. 调查方法与分析工具}

使用统一的调查问卷访问调查对象。问卷采用一 对一的询问填写方式, 由统一培训后的访问员逐题记 录。调查不包含对认知能力有严重障碍的老年人。数 据用 SPSS12.0 进行录入分析。

\section{4. 调查结果分析}

\section{1. 人口统计学特征}

在羊坊店街道约 $23 \%$ 的受访者为男性, 而在月 坛街道约 34\%为男性。

从年龄分布来看, 约 4\%（羊坊店）或 $11 \%$ (月坛)的受访者年龄在 80 岁及以上,约 $30 \%$ 至 $40 \%$ 的受访者年龄为 60-65 岁。

在这两个社区中, 约 $80 \%$ 的受访者都是已婚, $15 \%$ 至 $18 \%$ 的老年人丧偶, 不到 $1 \%$ 的人从未结过婚。

每个社区中不到 $10 \%$ 的受访者有过 6 年或以下 的学校教育, $30 \%$ 左右的参与者表明高中是他们的最 
Risk Analysis and Crisis Response in Big Data Era (RAC-16)

高学历。获得大学学历的受访者在羊坊店街道有 $23 \%$, 在月坛街道有 $31 \%$ 。

两个社区中大约有 $94 \%$ 的受访者都表示他们现 已退休。老年人被要求定义退休前所从事工作的类型 (或对那些仍然在工作的人来说是目前的工作)。羊 坊店和月坛分别有 $27 \%$ 和 $38 \%$ 的受访者回答是管理 者。18\% (羊坊店) 和 $14 \%$ (月坛) 的受访者回答 是体力劳动者。

\section{2. 生理健康状况}

老年人的总体生理健康状况是老年人安全和意 外伤害调查的重点之一。在这两个社区中, 约 $13 \%$ 的受访者表示, 在过去 7 天里, 他们的日常活动都曾 因身体健康问题受到限制。自我报告身体健康问题是 评估老年人身体状况的一种方式, 另一种方式是询问 老年人目前是否由于身体健康问题, 而在使用任何安 全设备。每个社区中都有约 $4 \%$ 的受访者表示, 他们 在过去 7 天使用过安全设备。

虽然使用安全设备是很明显的, 但不是所有人都 愿意承认自己的身体健康问题。为了克服这个问题, 我们要求受访者回答他们在上周是否发生过严重的 身体问题, 如走路、爬楼梯困难等。在羊坊店街道, 约 $22 \%$ 的老年人回答说, 他们行走困难。这个比例 在月坛街道较低。老年人的生理健康状况还可以用他 们的吃药频率来衡量。约 $23 \%$ 的受访者表示不因身 体健康问题吃药。羊坊店街道和月坛街道分别有 $10 \%$ 和 $14 \%$ 的受访者表示目前只吃中药。 $31 \%-26 \%$ 的受 访者表示只吃西药。3 6 \% 的受访者表示两种药都吃。 接下来我们要求受访者回答, 在过去 12 个月中, 他 们是否被专业医学人员诊断出问题。羊坊店街道和月 坛街道分别有 $41 \%$ 和 $44 \%$ 的受访者表示在过去 12 个月内被诊断过问题。约 $17 \%$ 和 $9 \%$ 的受访者 (分别 为羊坊店和月坛）表示, 在过去 12 个月内他们没看 过医生。

\section{3. 心理健康状况}

由于心理健康状况一般来说更难以诊断, 而且说 出自己的心理问题比说出身体健康问题更容易使受 访者产生耻辱感, 因此我们决定主要使用替代问题来 评估受访者的心理健康状况。

首先, 我们要求老年人回答, 他们的日常活动是 否由于心理健康问题而受到限制。在这两个社区中, $7 \%$ 的受访者表示, 在过去 7 天中, 他们的活动由于 心理健康问题受到过限制。接下来的两个问题是问受 访者, 是否和专业保健人士说过自己感觉悲伤或沮 丧。大约 5-6\%的老年人承认说过，约 $10 \%$ 的老年人
表示没有和专业医疗人士讨论过这些问题, 还有约 $85 \%$ 的老年人表示他们在过去 12 个月内没有这些感 觉。在这个情况下, 受访者被询问, 他们是否对一个 家庭成员或朋友说过自己感到伤心或沮丧。大约 $9 \%$ 的羊坊店街道受访者和 $12 \%$ 的月坛受访者表示曾与 亲近的人谈过这些问题。约 $23 \%$ 的羊坊店街道老年 人和 $67 \%$ 的月坛街道老年人表示, 他们不吃用于治 疗心理健康问题的药物。只吃中药、只吃西药或两种 药都吃的比例在两个社区中都低于 $10 \%$ 。悲伤、抑郁 或焦虑的感觉往往因寂寞加剧。不到 $10 \%$ 的受访者 表示, 他们在 3-7 天中与其他人的互动不到 1 个小时。 此外, 绝大多数受访者表示有一个家庭成员和自己住 的很近。

\section{4. 个体风险因素总结}

查看关于生理健康和心理健康问题的回答, 很明 显, 这些问题普遍存在于这两个街道的老年受访者 中。由于本调查的当务之急是超越对这些疾病的简单 说明, 于是使用其中一些回答作为风险因素。这些因 素可以对居住条件和安全设备的使用进行预测或解 释。我们确定个体风险因素, 所选因素和关联值（括 号内）如下:

一 年龄 (76 或以上)

一 婚姻状况 (从未结过婚、离婚、丧偶)

一 受教育情况 (6 年或以下)

一 由于生理健康状况而使日常活动受限 (是)

一 严重的行走困难、爬楼梯困难等 (是)

一 由于心理健康状况而使日常活动受限 (是)

一 对医生说过自己感到沮丧、悲伤 (是、否)

一 在过去 7 天中, 与他人互动少于 1 小时的天

数 $(3,4 、 5 、 6 、 7)$

一 最亲近的亲戚住的很远 (同个市、同个省、 不同省、国外、没有健在的亲戚)

在羊坊店街道, 约 $42 \%$ 的老年受访者没有风险 因素, 而在月坛街道约 36\%的受访者没有风险因素。 最高风险因素总和为 6 (在月坛街道小于 $0.2 \%$ )。除 了 0 以外, 两个社区中的多数情况是 1 个风险因素。

\section{5. 居住环境风险因素}

\subsection{1 客厅}

有关客厅条件的调查问题主要集中在地板的状 况上。这个问题是要求受访者回答地面的材质和大致 状况。这是个多项选择题, 可以让受访者有机会确认 所有给出的条件。选项分别为: 地面不平整、地面较 滑、地面较湿、木地板、防滑瓷砖地面、非防滑瓷砖 地面、裸露的混凝土地面或其他类型地面。在羊坊店 


\section{Risk Analysis and Crisis Response in Big Data Era (RAC-16)}

街道，约 14\%无风险因素的老年人和约 19\%有风险 因素的老年人表示家中客厅地面为木地板。月坛街道 的比例与羊坊店类似, $11 \%$ 无风险因素的老年受访者 和 $15 \%$ 有风险因素的老年受访者表示家中客厅地面 为木地板。在羊坊店街道中, $12 \%$ 无风险因素老年人 和 $15 \%$ 有风险因素老年人表示家中客厅地面为裸露 的混凝土。有趣的是, 在月坛街道, 无风险因素老年 人家中为混凝土地面的比例 (21\%) 高于有风险因素 的老年人 $(16 \%)$ 。

4.5.2 卧室

首先, 参与调查的老人被询问在他们的卧室地面 上是否有电线。在大部分老旧小区, 在起初设计时就 未预想到会大量使用电气设备。因此很多只能自己拉 电线, 这些电线就有绊倒人的危险。在这两个社区中, 表示地面上有电线的比例在有风险因素的老年人中 较高, 羊坊店街道 $42 \%$ (有风险) 和 $25 \%$ (无风险), 月坛街道 35\% (有风险) 和 30\% (无风险)。

接下来, 受访者被要求回答其卧室中的电源插座 是否有保护盖。在这个情况下, 羊坊店街道的受访者 中不存在差异 (无风险与有风险)。但是, 月坛街道 无风险因素的受访者表示有电源插座保护盖的比例 更高 $(70 \%$ 无风险与 $63 \%$ 有风险)。

4.5.3 卫生间

在报告的这一部分中调查的是卫生间中的安全 状况，包括地面状况以及防滑垫、扶手和电源插座保 护盖的使用。

在两个社区中，无风险因素的受访者（羊坊店 $77 \% \mathrm{vs} 72 \%$, 月坛 $74 \% \mathrm{vs} 70 \%$ ) 更倾向于表示在他们 所使用的卫生间中安装了电源插座保护盖。

关于在卫生间使用防滑垫的情况, 羊坊店街道有 风险因素和无风险因素的老年人中在回答这个问题 时存在差异。其中, $53 \%$ 无风险因素的老年人表示使 用防滑垫, 但是只有 $43 \%$ 有风险因素的老年人表示 在卫生间地面上使用防滑垫。月坛街道中这两组受访 者之间无明显差异，在 $43-44 \%$ 左右。

浴缸防滑垫的使用是另一个重要问题。羊坊店街 道有风险因素的受访者 $(26 \%)$ 和无风险因素的受访 者 $(26 \%)$ 都表示不太使用这种简单的安全设备。在 月坛街道, 有风险因素的老年人比无风险因素的老年 人更容易使用浴缸防滑垫 $(39 \% \mathrm{vs} 23 \%$ )。在浴缸的 安全性方面, 受访者被询问他们是否有扶手。和前面 两个情况一样, 羊坊店街道中有风险因素的老年人有 扶手的比例低于无风险因素的老年人 (33\%vs47\%)。 月坛街道的两组受访者中没有差异。

关于卫生间安全的其中一个问题是卫生间的地
面类型。当卫生间地面为非防滑的瓷砖地面时, 两组 受访者的回答差异很小。在这两个社区中，约 $20 \%$ 的受访老人表示家中是这种地面。羊坊店街道中无风 险因素和有风险因素受访组之间的差异基本可以忽 略, 但在月坛街道, 有风险因素的老年人家中更可能 为这种类型的地面一一更容易发生意外 ( $23 \%$ vs17\%)。

\subsection{4 厨房}

锋利的物体、潜在的火灾隐患、热的表面和湿滑 的地面是铌房中的几大潜在危险。在本调查中, 向老 年人询问了有关这些项目的问题。

在这两个社区中, 有风险因素受访者在不使用煤 气灶时关闭主燃气切断阀的可能性较高 (羊坊店 $24 \% \mathrm{vs} 19 \%$, 月坛 $23 \% \mathrm{vs} 21 \%$ )。

每个社区中都有 $5 \%$ 左右的受访者表明了不安全 厨具储存设备的危害。有风险因素与无风险因素受访 群之间的差异不明显。

有关在抽油烟机上方放置物品 (既是一个火灾隐 患, 也有坠落危险）方面, 在羊坊店街道, 约 $9 \%$ 有 风险因素的老年人表示在这个危险的位置放置物品, 无风险因素的老年人在这个问题上的比例为 $5 \%$ 左 右。月坛街道中这两组受访者之间的差异较为明显: 有风险因素的老年人为 $14 \%$, 无风险因素的老年人 为 $7 \%$ 。

关于厨房地面是否湿滑, 只有不到 $5 \%$ 的受访者 表示有这种危险情况的存在, 无风险因素老年人和有 风险因素老年人在这个问题上的回答差异不明显。

\section{6. 个体风险因素总结}

4.6.1 当前正在使用的安全设备

我们对羊坊店街道和月坛街道老年受访者关于 安全设备的使用情况进行了调查。首先, 在两个社区, 均有约 $10 \%$ 的老年受访者表示, 他们的住所配备了 火灾报警器。社区和两大群体 (无风险 vs 有风险) 之间的差异很小。在羊坊店街道，约 7\%的受访者表 示他们的住所有灭火器, 无风险因素老年人和有风险 因素老年人之间没有差异。在月坛街道，约 $19 \%$ 的 无风险因素老年人和约 $15 \%$ 的有风险因素老年人表 示在他们的公寓/房子里有灭火器。

在两个社区, 均有约 $59 \%$ 的受访者提到使用了 阳台上的防盗栏。社区和两大群体（无风险 vs 有风 险）之间的差异很小。约 $90 \%$ 的受访者表示家中有 防盗门。和此前一样, 社区和两大群体 (无风险 vs 有风险) 之间的差异很小。

在羊坊店街道, $76 \%$ 无风险因素的受访者和 $67 \%$ 有风险因素的受访者表示家中门上装有猫眼, 可 
以通过它们看到门外的人。在月坛街道, $59 \%$ 无风险 因素的受访者和 $66 \%$ 有风险因素的受访者表示家中 门上装有猫眼。

在羊坊店街道, $77 \%$ 无风险因素的老年人和 $69 \%$ 有风险因素的老年人表示家中有门禁电话 (在公 寓楼中很普遍)。在月坛街道, 约 $64 \%$ 的老年人表示 有门禁对话，两类风险人群之间没有差异。

\subsection{2 未来对安全设备的需求}

首先, 受访者被要求回答他们的厨房中是否需 要其他安全设备。在羊坊店街道, $22 \%$ 无风险因素的 老年受访者表示希望在他们的厨房中有更多的安全 设备, 而在有风险因素的老年受访者中这个比例只有 $13 \%$ 。在月坛街道, 约 $15 \%$ 的受访者希望在他们的 噼房中有其他安全设备。

在羊坊店街道参与问卷调查的老年人中, 需要其 他安全设备的比例约为 $10 \%$ 。在月坛街道, 约 $9 \%$ 的 受访者需要在卫生间安放其他安全设备。这两个社区 中两类风险人群之间的差异很小。

不到 $5 \%$ 的受访者表示需要在一般生活空间中放 置安全设备。不同社区和风险类型的老年人之间不存 在明显差异。

调查问卷要求受访者给出对住所以外 (社区中) 的其他安全设备的看法。在羊坊店街道, 约 $20 \%$ 的 老年人要求增加其他安全设备。两类风险人群之间的 差异不明显。在月坛街道, 这个比例低一点, 大约为 $16 \%$, 两类风险人群之间的差异可以忽略不计。

受访者被要求在高科技设施/有人机互动的高科 技设施/低科技设施中进行选择。受访者被要求对这 些选项按优先级排序 (图 1)。在这两个社区中 (与 风险因素无关), 绝大多数受访老年人更倾向于高科 技安全设备。

\begin{tabular}{|c|c|c|}
\hline 类型 & 月坛 & 羊坊店 \\
\hline 无风险-高科技设施 & $62.0 \%$ & $68.3 \%$ \\
\hline 无风险-人机互动的高科技设施 & $19.8 \%$ & $15.9 \%$ \\
\hline 无风险-低科技设施 & $18.2 \%$ & $15.9 \%$ \\
\hline 有风险-高科技设施 & $65.1 \%$ & $59.7 \%$ \\
\hline 有风险-人机互动的高科技设施 & $19.8 \%$ & $25.2 \%$ \\
\hline 有风险-低科技设施 & $15.1 \%$ & $15.0 \%$ \\
\hline
\end{tabular}

图 1 对高科技设施/人机互动的高科技设施/低科技设施的 需求情况排序

\section{5. 对策建议}

结果表明, 老年人普遍面临居家安全风险, 其中, 居家环境存在不安全隐患、缺乏使用必要的安全设备 是较为突出的问题, 另外相较于没有个体风险因素的
老年人而言, 有个体风险因素的老年人往往更容易忽 视自身的安全, 更少使用安全设备。

针对调查结果, 提出如下建议:

首先, 在北京的社区, 尤其老旧社区, 应加强适 老性改造, 减少造成老年人伤害的环境风险因素。 第二, 加强对老年人安全的相关培训和宣教工 作, 提高老年人安全意识, 增强老年人安全能力。 第三, 建议社区和老年人共同开展老年人居住环 境的隐患排查工作, 及时发现和消除环境安全隐患。 第四, 根据老年人需求, 研发科技水平高的安全 设备, 满足老年人日常生活的需要。

第五, 相对于无个体风险因素的老年人而言, 很 多有个体风险因素的老年人往往更容易忽视自身的 安全, 更少使用安全设备。因此, 在老年人安全工作 中, 个体风险高的老年人应该是重点关注的群体。

\section{参考文献}

[1] 王声湧. 伤害流行病学.人民卫生出版社, 北京, 2003 年.

[2] 席淑华, 马静, 卢根娣. 知信行理论干预社区高 龄独居老人意外伤害的效果.上海护理，2010, 10(003): 47-49.

[3] 瓮学清, 梁万年, 刘迎等. 北京市城市社区老年 人伤害疾病负担的研究. 疾病控制杂志, 2005 , 9(4): 302-305

[4] 于洪宇. 老年人跌倒问题的相关因素研究与预 防护理. 中国老年保健医学,2009, 7(1):85-88.

[5] 季淑风, 朱慧. 老年跌倒问题原因分析与预防. 中国康复理论与实践，13(1)，2007。

[6] 钟倩红, 林佩贤.广州市社区老年人伤害发生现 状及其影响因素分析. 现代预防医学, 37(1), 2010.

[7] Conwell Y. Suicide in later life: a review and recommendations for prevention. Suicide Life Threat Behav Suppl, 2001, (31):32-47.

[8] Kerber KA. Disequilibrium in older people: A prospective study. Phys Ther, 1997, 77(8):812819.

[9] 刘丽丹, 张衡. 老年人跌倒危险因素及其护理进 展. 现代临床护理, 11(11): 73-75，2012.

[10] Margda Waern, E Rubenwitz, B Runeson, etal Burden of illness and suicide in elderly people: case-control study. BMJ,8:1355, 2002.

[11] Nygaard HA . Falls and psychotropic drug consumption in long-term care residents: Is there an obvious association. Geront, 1998, 44(1):46-50.

[12] 王卓群, 杨静. 我国老年人跌倒的危险因素及预 防措施. 中国社区医师，2006，22(312):46-47.

[13] Wei Zhu, Qiuju You. High-rise Building Group Regional Fire Risk Assessment Model Based on AHP. Journal of Risk Analysis and Crisis Response, 2016, 6(1): 31-37. 